\title{
Can a mind have two time lines? Exploring space-time mapping in Mandarin and English speakers
}

\author{
Lynden K. Miles • Lucy Tan • Grant D. Noble • \\ Joanne Lumsden • C. Neil Macrae
}

Published online: 24 February 2011

(C) Psychonomic Society, Inc. 2011

\begin{abstract}
Spatial representations of time are a ubiquitous feature of human cognition. Nevertheless, interesting sociolinguistic variations exist with respect to where in space people locate temporal constructs. For instance, while in English time metaphorically flows horizontally, in Mandarin an additional vertical dimension is employed. Noting that the bilingual mind can flexibly accommodate multiple representations, the present work explored whether Mandarin-English bilinguals possess two mental time lines. Across two experiments, we demonstrated that Mandarin-English bilinguals do indeed employ both horizontal and vertical representations of time. Importantly, subtle variations to cultural context were seen to shape how these time lines were deployed.
\end{abstract}

Time and space are modes by which we think and not conditions in which we live.

Albert Einstein (1879-1955)

Einstein's psychological characterization of space and time as modes of thinking has, perhaps unsurprisingly, turned out to be remarkably astute. Punctuating verbal and nonverbal behavior alike, people tend to think about temporal constructs in spatial terms (Casasanto \& Boroditsky, 2008; Lakoff \& Johnson, 1980, 1999). In English, for example, people routinely talk of moving deadlines back, focusing on the week ahead, and leaving sad memories behind. Mirroring the use of such metaphors, patterns of linguistic processing,

L. K. Miles $(\bowtie) \cdot$ G. D. Noble $\cdot$ J. Lumsden • C. N. Macrae School of Psychology, University of Aberdeen, King's College, Aberdeen AB24 3FX Scotland, UK

e-mail: lynden.miles@abdn.ac.uk

L. Tan

University of Queensland,

Brisbane, Australia speech-related gestures, and movement dynamics also reveal that temporally relevant information is systematically ascribed to spatial locations (e.g., Kranjec, Cardillo, Schmidt, \& Chatterjee, 2010; Miles, Betka, Pendry, \& Macrae, 2010; Miles, Nind, \& Macrae, 2010; Núñez \& Sweetser, 2006). In short, the tendency to think about time in spatial terms can be seen to pervade cognition, language, and action.

While the grounding of abstract temporal information in concrete spatial representations may be a ubiquitous psychological phenomenon (Barsalou, 2008; Lakoff \& Johnson, 1980, 1999), there are nevertheless interesting sociolinguistic variations in the characteristics of this effect (see Casasanto, 2008, for an overview). Take, for example, contrasts between English and Mandarin. While spatiotemporal metaphors in English generally depict time as flowing along a horizontal plane, in Mandarin an additional vertical dimension (i.e., past $=$ up, future $=$ down) is also employed (Boroditsky, 2001; Chen, 2007; Scott, 1989; Yu, 1998). What is more, preliminary evidence suggests that these distinct characterizations of time may yield differences with respect to the manner in which temporal information is processed. In a seminal article, Boroditsky (2001, Experiment 1) reported that native English speakers respond to questions about time more rapidly when primed with information specifying a horizontal (vs. vertical) spatial relation, whereas native Mandarin speakers display the equivalent effect following vertical (vs. horizontal) primes (but see Chen 2007; January \& Kako, 2007). Similarly, Boroditsky, Fuhrman, and McCormick (2011) recently reported that Mandarin speakers were more likely to process temporal information as if it were structured vertically than were English speakers. ${ }^{1}$ What this suggests is that sociolin-

\footnotetext{
${ }^{1}$ This work was conducted independently of but concurrently with the present research.
} 
guistic conventions impact where, in space, temporal constructs are deemed to reside (see also Casasanto, 2008; Chan \& Bergen, 2005; Fuhrman \& Borodistsky, 2010; Núñez \& Sweetser, 2006; Tversky, Kugelmass, \& Winter, 1991).

Putative cultural differences in space-time mapping give rise to intriguing predictions for a specific group of individuals: Mandarin-English bilinguals. If sociolinguistic conventions shape where people mentally locate temporal constructs, what is the status of such mappings for those who speak two languages? Does the bilingual mind possess two mental time lines, each with the capacity to influence behavior, or do bilinguals rely on a single spatial characterization of time? Elsewhere, work on bilingualism has pointed to a degree of plasticity, with linguistic context moderating a range of basic psychological phenomena. For example, Danziger and Ward (2010) reported that bilingual Arab Israelis' maintain dual representations of the social world, such that their implicit attitudes toward Arabs and Jews differ depending on whether they are tested in Arabic or Hebrew. In much the same way, one might expect Mandarin-English bilinguals to maintain both horizontal (i.e., English) and vertical (i.e., Mandarin) representations of temporal information.

In a preliminary investigation of this possibility, Boroditsky (2001, Experiment 2) revealed that, within Mandarin-English bilinguals, the age of English acquisition was associated with the degree to which space-time mappings displayed a vertical (vs. horizontal) bias. Specifically, the younger the age of English acquisition, the greater the tendency to represent time horizontally. This finding hints at the operation of dual (i.e., horizontal and vertical) mental time lines to the extent that, for Mandarin speakers, learning English earlier in life increases the propensity to think about time horizontally, thereby reducing the relative magnitude of the verticality bias. As it turns out, however, attempts to corroborate Boroditsky's findings with either Mandarin (Chen, 2007) or English (January \& Kako, 2007) speakers have been largely unsuccessful, thereby calling into question the role that culture plays in space-time mapping. ${ }^{2}$

Revisiting this important theoretical issue, here, we report two experiments in which we investigated the hypothesis that being fluent in both Mandarin and English will lead to the operation of two distinct mental time lines, one arranged horizontally and another vertically. In Experiment 1, we sought to establish whether temporal constructs are spatially mapped according to sociolinguistic conventions by contrasting space-time mappings in English monolinguals and Mandarin-English bilinguals. In Experiment 2, we considered whether the cultural specificity

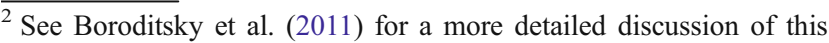
controversy.
}

of a task context influences how time is mapped to space among bilingual individuals.

\section{Experiment 1}

Experiment 1 required participants to categorize stimuli representing the past or future according to a spatial location. This approach has two main points of departure from the method employed by Boroditsky (2001, Experiment 1) and later by Chen (2007) and January and Kako (2007). First, we minimized the linguistic content of the task by requiring participants to classify visual stimuli-pictures of buildings from the past or future. By reducing linguistic demands simple carryover effects from, for instance, spatially oriented language can be controlled. Second, recent work indicates that movement may serve as an ideal vehicle for assessing spatial representations of temporal events (e.g., Miles, Betka, et al., 2010; Miles, Karpinska, Lumsden, \& Macrae, 2010; Miles, Nind, \& Macrae, 2010; Oliveri et al., 2009; Vallesi, Binns, \& Shallice, 2008). To this end, we required participants to make a movement to a (theoretically) spatially compatible or incompatible location when making past/future judgments.

\section{Method}

Participants and design Twenty-five Mandarin-English bilingual Singaporean residents (15 male, 10 female; mean age $=21.2$ years) and 19 monolingual (i.e., Englishspeaking) residents of Aberdeen, Scotland (7 male, 12 female; mean age $=19.7$ years) took part in the research. All of the bilingual participants used both Mandarin and English on a daily basis in both formal and informal settings, while none of the English speakers had any familiarity with Mandarin. The experiment had a 2 (orientation: horizontal or vertical) $\times 2$ (trial type: compatible or incompatible) repeated measures design.

Materials and procedure The target stimuli consisted of 22 color images $(890 \times 890 \mathrm{~mm})$ of buildings and cities, 11 representing the past (e.g., ancient ruins) and 11 representing the future (e.g., science fiction scenes). Examples are shown in Fig. 1a. Each testing session consisted of four blocks of trials: a compatible (past $=$ left, future $=$ right $)$ and an incompatible (past $=$ right, future $=$ left) horizontal block, as well as a compatible (past $=$ up, future $=$ down) and an incompatible (past $=$ down, future $=$ up) vertical block. Each image was presented twice within each block. Block order was counterbalanced across participants.

Participants were tested individually in English and were informed that a picture of a building/city would appear on the screen, which they were to categorize as being from the 
a
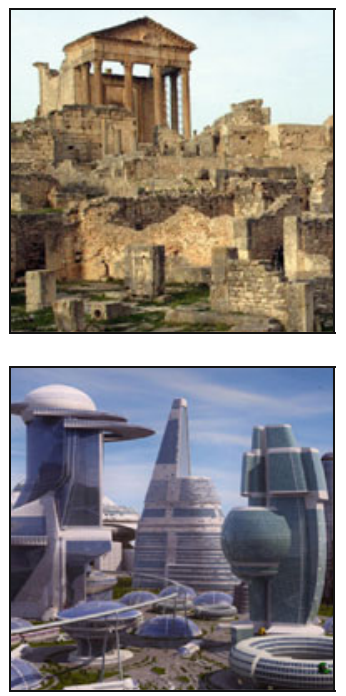

Bilingual Mandarin-English Speakers



Monolingual English Speakers

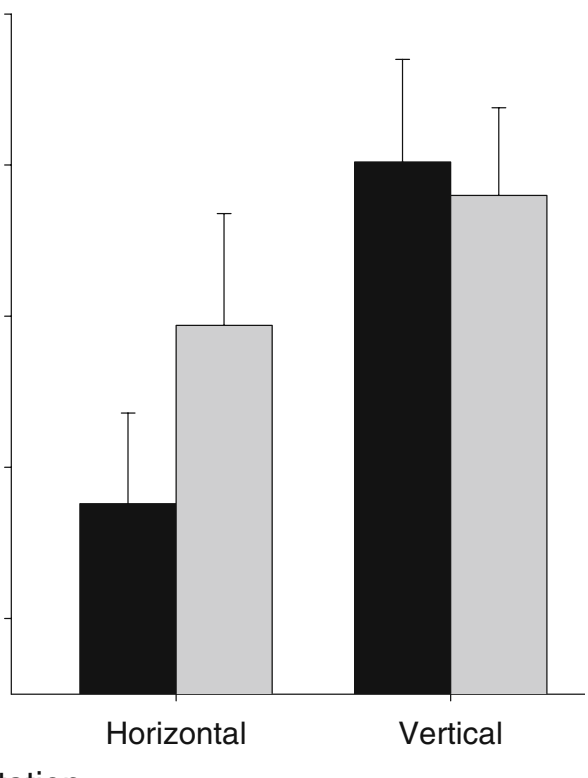

Fig. 1 a Examples of past and future targets used in Experiment 1 and $\mathbf{b}$ mean response time $(+1 S E M)$ as a function of orientation (horizontal vs. vertical) and trial type (compatible vs. incompatible) for the

past or future as quickly and accurately as possible. Participants were seated approximately $60 \mathrm{~cm}$ from the computer screen and, prior to each block of trials, received instructions specifying the response mapping (e.g., past $=$ up, future $=$ down) required for that block. To aid performance, response labels (i.e., "Past," "Future") were positioned at the appropriate parts of the screen (i.e., top and bottom edges for vertical blocks, left and right edges for horizontal blocks) and remained visible for the duration of each trial.

Participants indicated their responses using a standard numeric keypad. Each trial began with a centrally presented fixation cross, at which point the participants were required to press and hold the center key on the keypad (i.e., "5") with their right index finger. After a 1,000-ms delay, the target appeared centrally on the screen, prompting the participant to make their response by releasing the " 5 " key and pressing the appropriate response key, again with their right index finger. For blocks of horizontal trials, participants indicated their responses using the keys immediately to the left and right of the center key (i.e., "4" and " 6 "), while for blocks of vertical trials, the keys immediately above and below the center key were used (i.e., "2" and " 8 "). ${ }^{3}$ Importantly, this method of responding required a

\footnotetext{
$\overline{3}$ The keypad was mounted on an incline such that pressing the " 2 " or " 8 " key involved making a downward or an upward movement, respectively, but allowed the participant's hand position to remain relatively natural and comfortable.
}

Mandarin-English bilingual (left panel) and English monolingual (right panel) participants

movement to a position in space (e.g., on compatible trials in the horizontal condition, a past response is indicated by effecting a movement to the left).

\section{Results and discussion}

Trials on which participants made errors $(2.4 \%)$ or recorded a response latency that was more than 3 standard deviations from their mean $(0.7 \%)$ were omitted from the analyses. Mean response times (RTs) were compared using a 2 (group: Mandarin-English bilinguals vs. English monolinguals $) \times 2$ (orientation: horizontal vs. vertical) $\times 2$ (trial type: compatible vs. incompatible) mixed model analysis of variance (ANOVA) with repeated measures on the final two factors. This revealed main effects of orientation, $F(1,42)=20.03, p<.01, \eta_{\mathrm{p}}{ }^{2}=$ .32 , and trial type, $F(1,42)=6.96, p<.05, \eta_{\mathrm{p}}{ }^{2}=.14$, which were qualified by a group $\times$ orientation $\times$ trial type interaction, $F(1,42)=4.90, p<.05, \eta_{\mathrm{p}}{ }^{2}=.10$ (see Fig. 1b)

To examine the three-way interaction more closely, follow-up 2 (orientation: horizontal vs. vertical) $\times 2$ (trial type: compatible vs. incompatible) repeated measures ANOVAs were conducted for each group separately. For the Mandarin-English bilinguals, this revealed main effects of orientation, $F(1,24)=4.78, p<.05, \eta_{\mathrm{p}}{ }^{2}=.17$, and trial type, $F(1,24)=5.76, p<.05, \eta_{\mathrm{p}}{ }^{2}=.19$. However, no orientation $\times$ trial type interaction was observed, $F<0.5$. Mandarin-English bilinguals responded faster on compat- 
ible $(M=736 \mathrm{~ms})$ than on incompatible $(M=772 \mathrm{~ms})$ trials, regardless of the orientation (i.e., horizontal or vertical) of the time line. In comparison, English monolinguals also showed a main effect of orientation, $F(1,18)=$ 15.49, $p<.01, \eta_{\mathrm{p}}{ }^{2}=.46$, but this was qualified by an orientation $\times$ trial type interaction, $F(1,18)=5.07, p<.05$, $\eta_{\mathrm{p}}^{2}=.22$. Post hoc testing (Tukey $a, p<.05$ ) revealed a significant difference between RTs for the compatible and incompatible horizontal trials but no such difference for the vertical trials. Thus, monolingual English speakers displayed only the operation of a horizontal mental time line.

Taken together, these results reveal a distinct contrast between the two groups. The Mandarin-English bilingual participants were faster to classify items representing the past and future according to a sociolinguistically compatible (vs. incompatible) location, regardless of whether they did so along a horizontal or a vertical axis. In contrast, the monolingual English-speaking participants showed this effect only when organizing time horizontally; for this group, the past and future were not systematically associated with vertical position in space. These findings support and extend those reported by Boroditsky and colleagues (Boroditsky, 2001; Boroditsky et al., 2011). Using a somewhat different procedure, here, we demonstrate not only that bilingual Mandarin-English speakers possess a vertical space-time mapping commensurate with sociolinguistic conventions within Mandarin, but also that these same individuals map time to space along a horizontal axis, consistent with the effect shown by monolingual English speakers. As such, it appears that the language one speaks can indeed influence how one thinks about time, such that bilinguals possess two mental time lines.

\section{Experiment 2}

Beyond demonstrating a systematic link between language and cognition with respect to the manner in which temporal information is spatially encoded, the results of Experiment 1 give rise to an important question. If Mandarin-English bilinguals possess two mental time lines, each consistent with prevailing sociolinguistic conventions, how are they deployed in everyday settings? In particular, is one specific mapping routinely preferred over the other, or do contextual factors dictate how one thinks about time?

To address this question, we adapted a procedure used by Tversky et al. (1991) whereby participants were asked to arrange photographs into a temporally ordered sequence. Two sets of photographs were employed, each of which consisted of three images of the same individual at different ages (see Fig. 2a). Importantly, one set depicted Jet Li, a prominent Chinese actor, while the other depicted Brad Pitt, a famous American actor. In this way, we were able to manipulate a culturally relevant aspect of the task context (i.e., the cultural identity of the target) without explicitly drawing attention to pertinent linguistic distinctions between Mandarin and English.

\section{Method}

Participants and design Thirty-two Mandarin-English bilingual Singaporean residents (19 male, 12 female; mean age $=23.1$ years) took part in the experiment. As in Experiment 1, all the participants used both Mandarin and English on a daily basis. The procedure had a single-factor (target: Jet $\mathrm{Li}$ or Brad Pitt) withinparticipants design. Target order was counterbalanced across participants.

Materials and procedure The photographs were printed in color on $10 \times 10 \mathrm{~cm}$ cards and depicted the targets at three distinct ages. In the final image in each sequence (i.e., the oldest), the target was wearing prosthetic makeup for a role in which he played an elderly person. All the participants were tested individually in English. On each trial, the experimenter initially placed the middle photograph in the sequence in the center of a $30 \times 30 \mathrm{~cm}$ board and handed the remaining two photographs to the participant, along with an instruction to place these pictures on the board (see Tversky et al., 1991). The experimenter recorded both the orientation (horizontal, vertical, diagonal) and direction (e.g., left to right) of each sequence the participant created.

\section{Results and discussion}

Participants exclusively used the horizontal and vertical axes when creating the temporal sequences. Moreover, only sequences temporally ordered from left to right and from top to bottom were produced. To assess whether participants arranged the photographs differently as a function of the cultural identity of the target, the proportion of vertically arranged sequences was compared between the Jet Li and Brad Pitt trials, using Cochran's $Q$ test. This revealed a significant effect, $Q(1)=4.76, p<.05$, such that participants were more likely to arrange Jet $\mathrm{Li}$, the Chinese target, vertically than they were Brad Pitt, the American target (see Fig. 2b). ${ }^{4}$

This finding provides further evidence for the operation of both horizontal and vertical mental time lines in the minds of

\footnotetext{
$\overline{{ }^{4} \text { An additional }} 20$ monolingual English speakers also performed this task. Nineteen participants ordered the sequences horizontally (left to right), regardless of the target. One participant arranged Brad Pitt horizontally (left to right) and Jet Li vertically (top to bottom).
} 
a
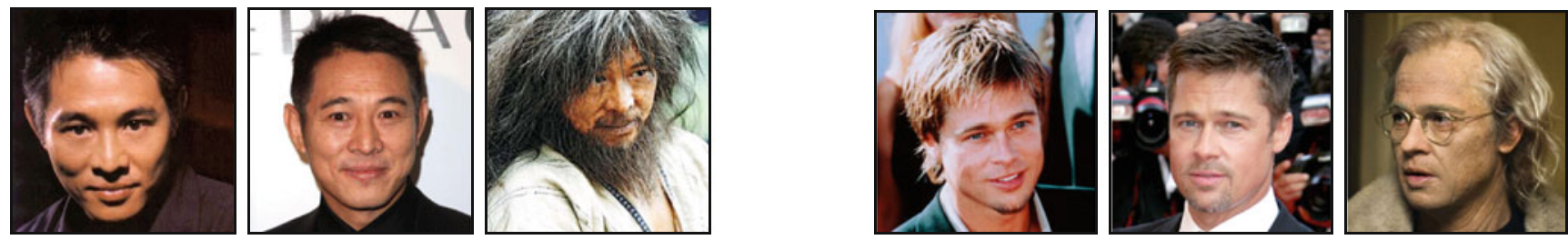

b

Jet Li

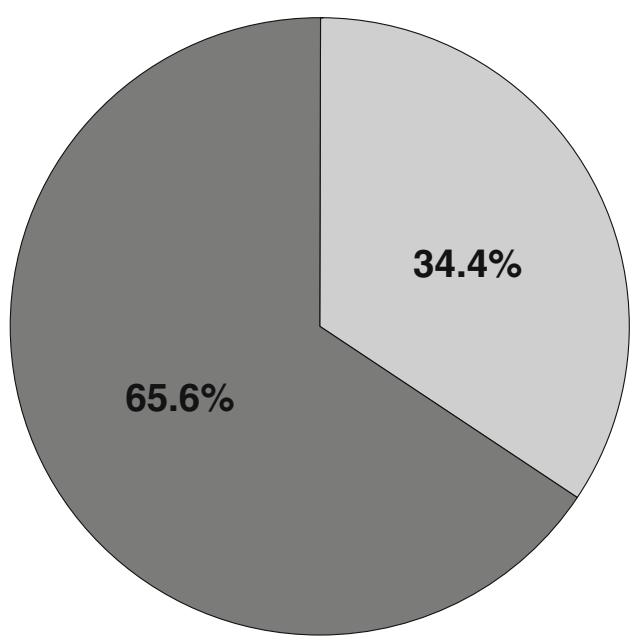

Horizontal Vertical

\section{Brad Pitt}

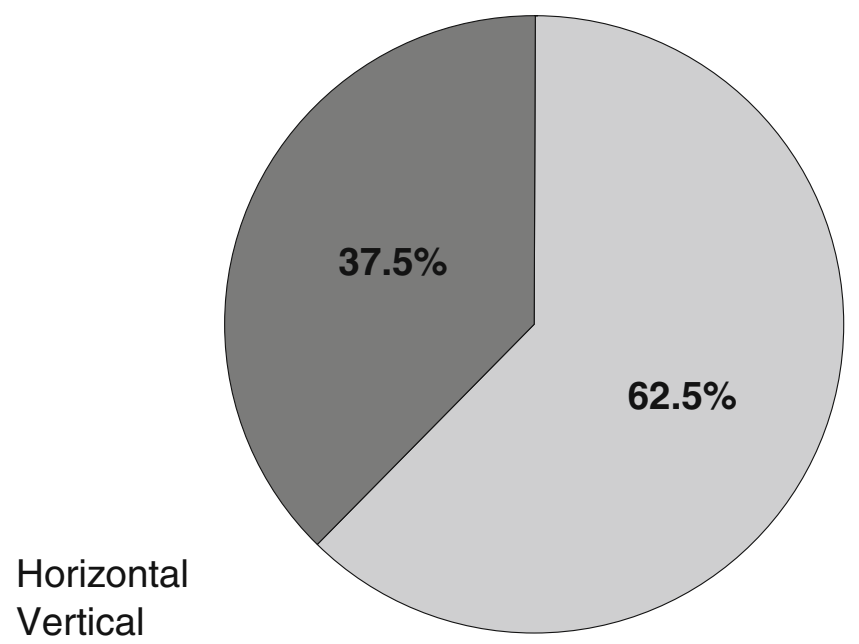

Fig. 2 a Sequences of Jet Li and Brad Pitt photographs used in Experiment 2 and $\mathbf{b}$ the proportion of horizontally and vertically arranged sequences as a function of target

Mandarin-English bilinguals. Moreover, the results reveal that subtly highlighting an Eastern cultural context (via the Jet Li photographs) biased these participants to use a vertical time-space mapping, while promoting a Western context (via the Brad Pitt photographs) prompted the use of the horizontal axis when they thought about time.

\section{General discussion}

Across two experiments - each with varied task requirements-Mandarin-English bilinguals were shown to employ both horizontal and vertical representations of time. Distinctions between the way time is characterized in Mandarin and English were reflected in the manner in which participants spatially mapped temporal constructs. What is more, these effects emerged in tasks in which linguistic content was minimized. In this way, the language(s) the participants spoke was seen to influence how they thought about time, even when such thinking was revealed in primarily nonlinguistic contexts. Together, these experiments demonstrate that multiple time lines exist in the minds of Mandarin-English bilinguals, each with the capacity to shape cognition and behavior (Boroditsky, 2001, Boroditsky et al., 2011).
Aside from establishing that Mandarin-English bilinguals possess both vertical and horizontal mental time lines, the present results also have important implications for understanding quite how these multiple mappings are employed. Although participants in Experiment 2 performed what was ostensibly the same task (i.e., arranging a temporal sequence) twice, they did so in markedly different ways depending on culturally specific characteristics of the target (i.e., Jet Li vs. Brad Pitt). What this suggests is that heightening the salience of one sociolinguistic context over the other (i.e., Mandarin vs. English) resulted in participants' spontaneously adopting a spatial representation of time consistent with prevailing norms within that language. This finding adds further weight to Boroditsky's (2001; Boroditsky et al., 2011) supposition that distinctions between Mandarin and English shape spatiotemporal thinking, but it also raises an important question regarding the cognitive status of the "unused" mental time line. In particular, if an individual possesses both horizontally and vertically oriented spatial mappings of time, do these time lines coexist in an independent manner, or do they compete such that if one is selected (i.e., deployed), the other is inhibited? Understanding the cognitive dynamics of space-time mappings in the 
bilingual mind remains an important task for future research.

It remains unclear, however, precisely which distinctions between Mandarin and English drive the present effects (cf. Boroditsky, 2001; Boroditsky et al., 2011). For instance, while Mandarin contains metaphors that depict a vertical passage of time (Boroditsky, 2001; Chen, 2007; Scott, 1989; Yu, 1998), there are no equivalent mediolateral (i.e., left-right) temporal metaphors in English (Santiago, Lupiáñez, Pérez, \& Funes, 2007). To this end, it has been argued that other conventions (i.e., reading/writing direction) play a prominent role in shaping the cognitive mapping of time along the mediolateral axis (Chan \& Bergen, 2005; Santiago et al., 2007; Tversky et al., 1991). However, this factor may not provide a sufficient explanation for the present effects either, since both English and Mandarin (i.e., simplified Chinese version) are written horizontally in Singapore. ${ }^{5}$ In this respect, it appears that multiple aspects of sociolinguistic context have the potential to influence the structure of spacetime mappings (Miles, Betka, et al., 2010). Distinguishing between the impact of the conventions (e.g., writing direction) and contents (e.g., metaphors) inherent to the variations between distinct linguistic contexts will help elucidate this issue.

In a more general sense, the present findings are also consistent with a growing body of research demonstrating that sociolinguistic context can shape the manner in which bilingual individuals think about their world (e.g., Danziger \& Ward, 2010; Marian \& Kaushanskya, 2007; Marian \& Neisser, 2000). Studies of this kind have been taken to support the Sapir-Whorf linguistic relativity hypothesis (Whorf, 1956), which suggests that language is the primary determinant of thoughts and behavior. Indeed, the present results could also lead to such a conclusion, given that bilingualism triggered the operation of two distinct mental time lines. As January and Kako (2007) have pointed out, however, identifying relationships between the language(s) one speaks and differences in cognition does not necessarily isolate language per se as the causal influence in shaping thought (Boroditsky et al., 2011; Casasanto, 2008). Until alternative explanatory pathways (e.g., cultural convention) can be ruled out and definitive mechanisms elucidated, key questions will remain as to the precise status of the relationship between linguistic experience and cognitive operations. What is clear, however, is that the mind can possess two distinct mental time lines or, in other words, two modes by which time is mapped to space.


text/characters is a feature Singaporean Mandarin speakers are familiar with and are exposed to in their everyday lives.
}

Acknowledgements The authors are extremely grateful to the Tan family, whose generous hospitality and assistance made this research possible. L.K.M. was supported by a Research Councils of the U.K. Academic Fellowship, and C.N.M. was supported by a Royal SocietyWolfson Fellowship.

\section{References}

Barsalou, L. W. (2008). Grounded cognition. Annual Review of Psychology, 59, 617-645.

Boroditsky, L. (2001). Does language shape thought?: Mandarin and English speakers' conceptions of time. Cognitive Psychology, 43, $1-22$.

Boroditsky, L., Fuhrman, O., \& McCormick, K. (2011). Do English and Mandarin speakers think about time differently? Cognition, $118,123-129$.

Casasanto, D. (2008). Who's afraid of the big bad Whorf? Crosslinguistic differences in temporal language and thought. Language Learning, 58, 63-79.

Casasanto, D., \& Boroditsky, L. (2008). Time in mind: Using space to think about time. Cognition, 106, 579-593.

Chan, T. T., \& Bergen, B. (2005). Writing direction influences spatial cognition. In B. Bara, L. W. Barsalou, \& M. Bucciarelli (Eds.), Proceedings of the 27th Annual Conference of the Cognitive Science Society (pp. 412-417). Mahwah, NJ: Erlbaum.

Chen, J.-Y. (2007). Do Chinese and English speakers think about time differently? Failure of replicating Boroditsky (2001). Cognition, 104, 427-436.

Danziger, S., \& Ward, R. (2010). Language changes implicit associations between ethnic groups and evaluation in bilinguals. Psychological Science, 21, 799-800.

Fuhrman, O., \& Boroditsky, L. (2010). Cross-cultural differences in mental representations of time: Evidence from an implicit nonlinguistic task. Cognitive Science, 34, 1430-1451.

January, D., \& Kako, E. (2007). Re-evaluating evidence for linguistic relativity: Reply to Boroditsky (2001). Cognition, 104, 417-426.

Kranjec, A., Cardillo, E. R., Schmidt, G. L., \& Chatterjee, A. (2010). Prescribed spatial prepositions influence how we think about time. Cognition, 114, 111-116.

Lakoff, G., \& Johnson, M. (1980). Metaphors we live by. Chicago: University of Chicago Press.

Lakoff, G., \& Johnson, M. (1999). Philosophy in the flesh. New York: Basic Books.

Marian, V., \& Kaushanskya, M. (2007). Language guides memory context. Psychonomic Bulletin \& Review, 14, 925-933.

Marian, V., \& Neisser, U. (2000). Language-dependent recall of autobiographical memories. Journal of Experimental Psychology: General, 129, 361-368.

Miles, L. K., Betka, E., Pendry, L. F., \& Macrae, C. N. (2010). Mapping temporal constructs: Actions reveal that time is a place. Quarterly Journal of Experimental Psychology, 63, 2113-2119.

Miles, L. K., Karpinska, K., Lumsden, J., \& Macrae, C. N. (2010). The meandering mind: Vection and mental time travel. PLOS ONE, 5, e10825. doi:10.1371/journal.pone.0010825

Miles, L. K., Nind, L. K., \& Macrae, C. N. (2010). Moving through time. Psychological Science, 21, 222-223.

Núñez, R. E., \& Sweetser, E. (2006). With the future behind them: Convergent evidence from Aymara language and gesture in the crosslinguistic comparison of spatial construals of time. Cognitive Science, 30, 1-49.

Oliveri, M., Bonni, S., Turriziani, P., Koch, G., Lo Gerfo, E., Torriero, S., et al. (2009). Motor and linguistic linking of space and time in the cerebellum. PloS ONE, 4, e7933. doi:10.1371/journal. pone. 0007933 
Santiago, J., Lupiáñez, J., Pérez, E., \& Funes, M. J. (2007). Time (also) flies from left to right. Psychonomic Bulletin \& Review, 14, 512-516.

Scott, A. (1989). The vertical dimension and time in Mandarin. Australian Journal of Linguistics, 9, 295-314.

Tversky, B., Kugelmass, S., \& Winter, A. (1991). Cross-cultural and developmental trends in graphic productions. Cognitive Psychology, $23,515-557$
Vallesi, A., Binns, M. A., \& Shallice, T. (2008). An effect of spatialtemporal association of response codes: Understanding the cognitive representations of time. Cognition, 107, 501-527.

Whorf, B. L. (1956). Language, thought, and reality. Cambridge, MA: Technology Press of MIT.

$\mathrm{Yu}, \mathrm{N}$. (1998). The contemporary theory of metaphor: A perspective from Chinese. Amsterdam: Benjamins. 